\title{
Sequence analysis shows that Lifeguard belongs to a new evolutionarily conserved cytoprotective family
}

\author{
KERSTIN REIMERS, CLAUDIA Y.-U.CHOI, EDDY MAU-THEK and PETER M. VOGT \\ Department for Plastic, Hand and Reconstructive Surgery, Medical School Hannover, \\ Podbielskistrasse 380, 30659 Hannover, Germany
}

Received March 2, 2006; Accepted May 8, 2006

\begin{abstract}
Cellular sensitivity to apoptotic stimuli is determined by several regulatory proteins. The biological and biomedical impact of these regulatory proteins is of fundamental importance for understanding and controlling apoptotic processes. We used a bioinformatic approach to characterise the antiapoptotic protein Lifeguard (LFG). LFG is an evolutionarily well-conserved protein with homologues in many species. Due to its hydrophobic nature it is predicted to reside in cellular membranes, namely the endoplasmatic reticulum and the plasma membrane, with seven transmembrane spanners and a small cytoplasmic domain. The consensus motif of a protein family with unknown function UPF0005 was found in the C-terminus. The structure of Lifeguard resembles the antiapoptotic protein Bax Inhibitor-1 (BI-1). Concordantly, it was shown that Bax co-immunoprecipitates with LFG. Our results indicate that LFG belongs to a new cytoprotective family with evolutionarily conserved functions in the prevention of programmed cell death.
\end{abstract}

\section{Introduction}

Apoptosis is a cellular suicide mechanism which protects multicellular organisms against unwanted or dangerous cells. This process, which is also termed programmed cell death, can be triggered on extrinsic pathways by certain receptors on the cellular surface called death receptors or induced intrinsically by intracellular proteins such as apoptotic protease-activating factor 1 (Apaf-1), whose activation follows the release of cytochrome c from damaged mitochondria. Other pathways include the granzyme B-mediated cleavage of caspases and response to stress in the endoplasmatic reticulum (ER).

Numerous cellular and viral inhibitors of apoptosis have been described. Members of the Bcl-2 family interfere with the mitochondrial pathway (1), while members of the IAP

Correspondence to: Dr Kerstin Reimers, Klinik für Plastische, Hand- und Wiederherstellungschirurgie, Medizinische Hochschule Hannover, Podbielskistrasse 380, D-30659 Hannover, Germany

E-mail: reimers.kerstin@mh-hannover.de

Key words: protein family, Bax-inhibitor, programmed cell death, bioinformatics, apoptosis inhibitor family suppress apoptosis through their interaction with caspases (2). cFLIP ${ }_{\mathrm{L}}$ and its cellular and viral homologues intervene ambiguously in death receptor-induced apoptotic signalling. Being a structural but enzymatically inactive homologue of Caspase 8 , formation of the death complex seems to be inhibited (3-5).

Lifeguard has been described as a new anti-apoptotic protein protecting cells against Fas mediated cell death (6). Homology search in the genetic databases showed that the same protein has been identified before as neural membrane protein 35 (NMP 35) $(7,8)$. However, while Northern blot analysis proved that LFG is highly expressed in the brain, it was also found in many other tissues. Transient expression of LFG in HeLa cells suggested that it is membrane associated and binds to Fas. It was not possible to show any downregulation of Fas or interference with the formation of the death complex, however (6). As a consequence, the molecular mechanism of apoptosis regulation executed by LFG remains to be further elucidated.

We used a bioinformatical approach to characterise the proteomical properties of LFG. Sequence analysis showed that LFG is a evolutionarily conserved protein containing seven transmembrane domains. Its localisation was predicted to the ER and the plasma membrane, respectively. At the Cterminus we identified a conserved domain characteristic of a new protein family with Bax inhibitory functions. In coimmunoprecipitations we demonstrated that Lifeguard interacts with Bax. Our results showed that Lifeguard is a member of a family of transmembrane proteins with antiapoptotic functions.

\section{Materials and methods}

Database search. Protein sequences were retrieved from GenBank at the National Center for Biotechnology Information (9). The accession numbers of the protein sequences analysed in this study are as follows : H. sapiens (NP_036438.2), $M$. musculus (NP_082500.2), R. norvegicus (NP_653357.1), G. gallus (XP_424507.1), D. melanogaster (NP_610824.1), C. elegans (NP_741597.1), and A.thaliana (NP_193209.2).

Sequence analysis. The degree of sequence identity was determined using ALIGN (http://workbench.sdsc.edu/) and the alignment scores at HomoloGene with BLOSOM62 matrix and expect value calculation based on the $\mathrm{nr}$ division of GenBank. Multiple sequence alignment was performed using 

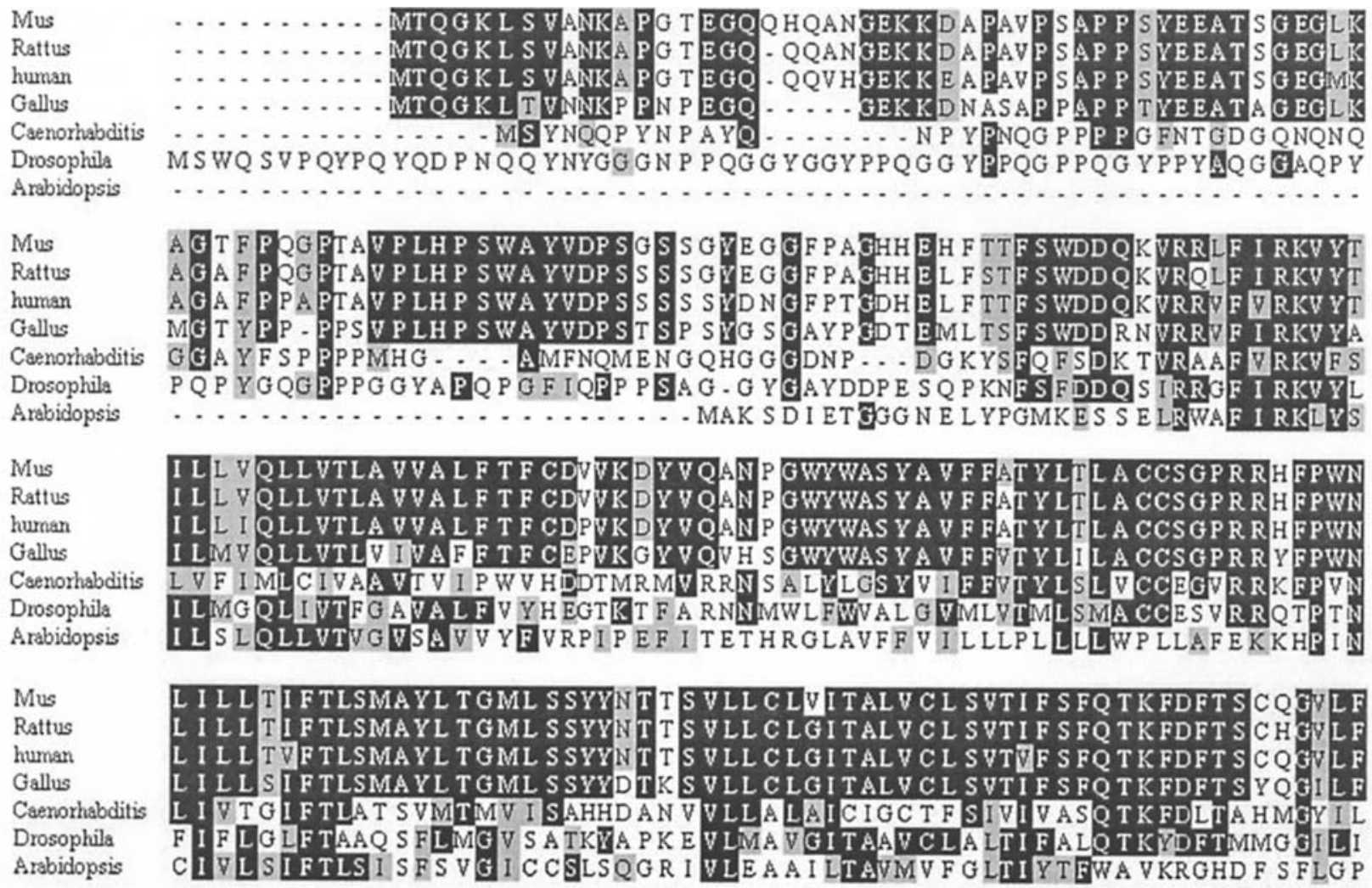

\begin{tabular}{|c|c|}
\hline s & 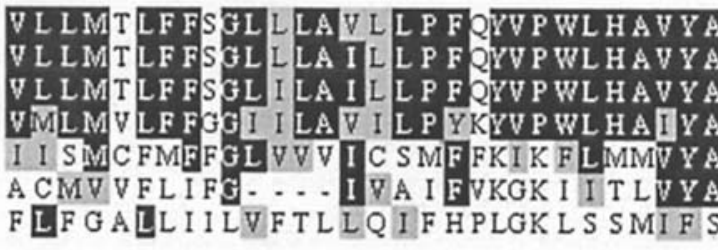 \\
\hline $\begin{array}{l}\text { Ius } \\
\text { attus } \\
\text { Iman } \\
\text { allus } \\
\text { aenorhabditis } \\
\text { rosophila }\end{array}$ & 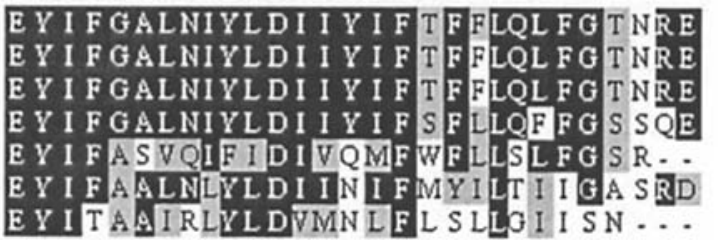 \\
\hline
\end{tabular}

Figure 1. Protein alignments of LFG. Protein sequences of human, mouse, rat, G. gallus, D. melanogaster, C. elegans and Arabidopsis were aligned using Clustal W (MegAlign). Shading of the amino acids was performed with the BoxShade program. Identical amino acids are shaded in black, grey colour indicates conservative changes.

the ClustalW program within the DNAstar MegAlign sequence analysis software package (DNAstar Inc., Madison, WI). A printable version was generated with BoxShade (http:// bioweb.pasteur.fr/seqanal/interfaces/boxshade.html). The primary amino acid sequence of human LFG was analysed by several programs to predict the transmembrane region and topology (TOPPRED2, TMPRED, TMHMM, HMMTOP and SMART) as well as the localisation of LFG (PSORT II, SignalP and TargetP). A Kyte and Doolittle hydropathic plot (10) was generated with ProtScale (http://www.expasy.org/ cgi-bin/protscale.pl) with Kyte and Doolittle option. Pfam (http://pfam.wustl.edu/) and SMART domain analysis programs were used to predict the domain architecture of LFG. We used ScanProsite (http:www.expasy.org/tools/ scanprosite/PROSITE) and NCBI RPS-BLAST for conserved domain database screening as described elsewhere (11-15). Posttranslational modifications were predicted with various programs at the EXPASY server and interpreted in conformity with the results of the topology prediction. Coiledcoil (http:// npsa-pbil.ibcp.fr/cgi-bin/npsa_automat.pl?page=/NPSA/ npsa_server.html) was used to screen for possible coiled coils.

cDNA constructs and transfection of HepG2 cells. cDNA encoding full-length LFG was cloned from a human cDNA library (lym12, Invitrogen, Carlsbad, CA). Specific primers were generated after the published sequence (GenBank: NM_012306). The complete coding sequence was cloned into the expression vector pcDNA5/FRT/V5-TOPO (Invitrogen). For transfection HepG2 cells were seeded in 6-well plates and grown overnight to subconfluency. The transfection reagent Fugene6 (Roche Molecular Biochemicals, Mannheim) was mixed with serum-free culture medium and incubated for $5 \mathrm{~min}$ at room temperature. Plasmid DNA was added at 


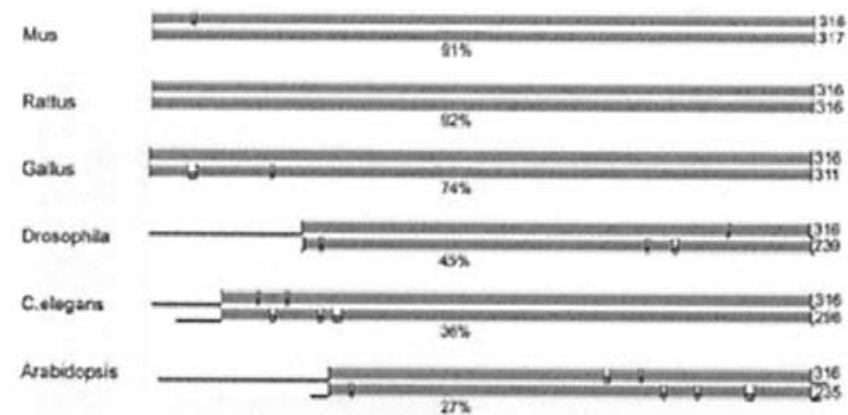

Figure 2. Schematic representation of the LFG homologues compared to the human sequence according to a local alignment with BLAST2. The degree of amino acid similarity ( $\%$ identity $\mathrm{x} \%$ positive) and homologous regions between LFG and other representative proteins of animal and plant origin are presented as graphical bars. Regions of high similarity are indicated as grey bars, gaps are marked by brackets. The number of amino acid residues of the represented protein is given at the right.

$3.75 \mu \mathrm{g} /$ well and incubated for $15 \mathrm{~min}$ at room temperature. Cells were incubated for $2 \mathrm{~h}$ with the transfection mixture and then fed.

RT-PCR and LFG expression. To confirm LFG expression in the used cell lines, $2 \mu \mathrm{g}$ total RNA isolated by RNAII (Macherey-Nagel, Düren, Germany) were reverse transcribed in a reaction containing $0.25 \mathrm{mM}$ dNTP-mix, $2 \mu \mathrm{g}$ random hexamers, $20 \mathrm{U}$ recombinant StratascriptII (Stratagene, La Jolla, CA) with 1X Stratascript buffer supplied by the manufacturer according to the instructions. Of the reaction mixture, $1 \mu 1$ was used in polymerase chain reaction with the specific primers. As a reaction control primers for $18 \mathrm{~S}$ rRNA were used. The amplification products were analysed on a $2 \%$ agarose gel supplemented with EtdBr.

Co-immunoprecipitation. Dynabeads ${ }^{\circledR}$ ProteinG (Dynal Biotech Inc., Brown Deer, WI) were washed according to the manufacturer's instructions and finally resuspended in $0.5 \mathrm{ml}$ $0.1 \mathrm{M} \mathrm{Na}$-citrat pH 5.0. Samples were added and incubated for up to $40 \mathrm{~min}$ at room temperature. Test tubes were placed in a magnet reck and the supernatant was removed. After three washing steps with $0.5 \mathrm{ml} 0.1 \mathrm{M} \mathrm{Na}$-citrat $\mathrm{pH} 5.0$ protein complexes were eluted two times with $30 \mu 10.1 \mathrm{M} \mathrm{Na-citrate}$ buffer $\mathrm{pH}$ 2.0. Samples were subjected to SDS-PAGE followed by Western blotting. All critical experiments were repeated at least three times. Representative results are shown in Figs.

\section{Results}

LFG is an evolutionary conserved protein. Protein sequences of seven different species were extracted from GenBank and arranged in multiple alignments. Analysis using the ClustalW algorithm revealed that LFG homologues share a high level of identity between the analysed species (Fig. 1). Local alignment with BLAST2 program of NCBI was used to compare the LFG sequences to the human homologue (Fig. 2). We found $91 \%$ identity for mouse and $92 \%$ for rat, respectively. Identity with the sequence of Gallus was 74\%; $45 \%$ for Drosophila and $36 \%$ for Caenorhabditis. An LFG homologue was also identified in Arabidopsis with $27 \%$ identity to the human
A

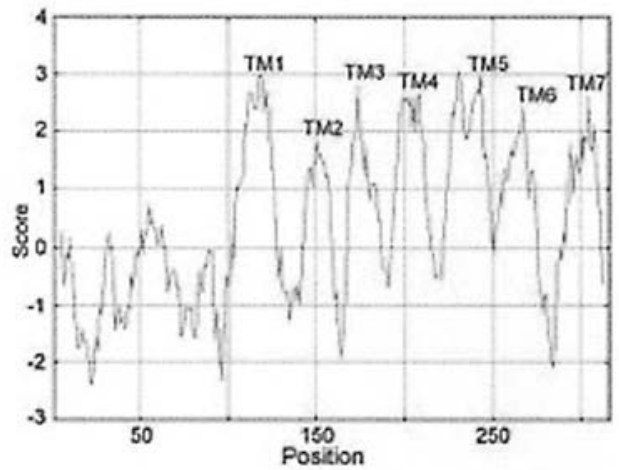

B

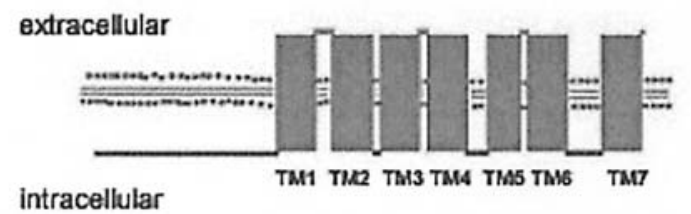

Figure 3. Domain architecture of LFG. (A) Hydrophobicity plot according to the Kyte and Doolittle method using a window size of 13 amino acids. The putative TM segments are indicated. (B) A proposed membrane spanning topology of LFG showing the transmembrane domain organisation.

sequence. The similarity was calculated based on the fulllength proteins of the non-human species compared to the human sequence: $86 \%$ for mouse, $87 \%$ for rat, $63 \%$ for chicken, 29\% for Drosophila and 20\% for Caenorhabditis, Arabidopsis had $13 \%$. The rates of similarity were nearly the same over the entire length of the analysed proteins except for some divergence at the N-termini (Fig. 2).

Amino acid sequence analysis of LFG reveals a multi-TM protein. It has been suggested that LFG is a membrane associated protein (6). Computer assisted analysis of the human LFG amino acid sequence indicated a number of putative TM domains. As expected from the overall amino acid composition, the Kyte and Doolittle hydrophobicity plot for LFG showed a highly hydrophobic pattern with seven clear spikes indicating a polytopic membrane protein (Fig. 3A). To analyse the number of TM domains and the possible topology various programs (TOPPRED2, TMPRED, TMHMM, HMMTOP and SMART) were used. All of the methods independently offered very clear predictions, assuming 7 TM spanners (Figs. 3B and 4). The TM domains were positioned according to hydropathy values and minimal charge density. A schematic model of the membrane spanning domains is depicted in Fig. 3B. Presumably, LFG belongs to the membrane proteins type $3 \mathrm{a}$. Another putative TM was found at the $\mathrm{N}$-terminus of the human sequence, but showed a low probability score.

Taking into concern the hydrophobic nature of the protein SignalP was used to look for N-terminal signalling peptides, but they were not detected. Subcellular localisation was predicted using PSORT II, SignalP and TargetP. The highest probabilities resulted for a localisation in the ER and in the plasma membrane.

In a search for possible posttranslational modifications different programs at the EXPASY server were used. As a 


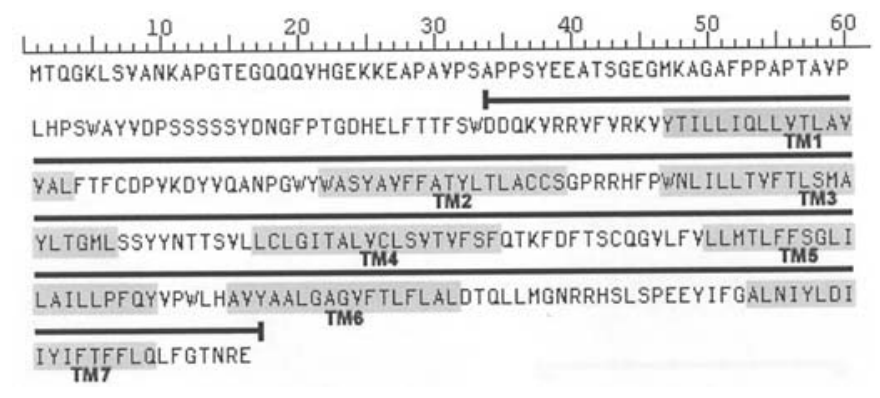

Figure 4. Amino acid sequence of human LFG. TM domains are depicted with grey boxes. A black bar indicates the UPF0005 family motif.

A

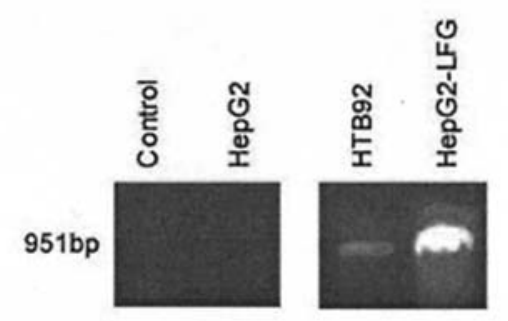

B

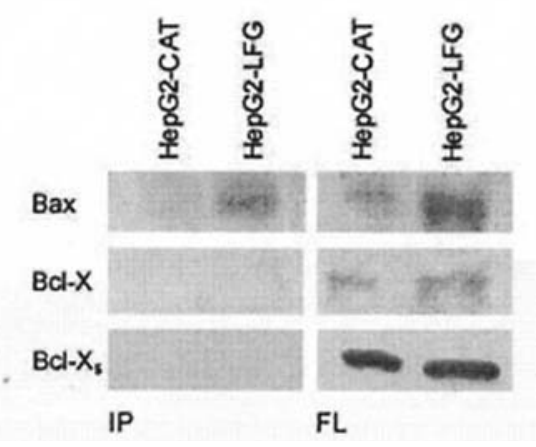

Figure 5. (A) RT-PCR analysis for LFG expression. cDNA was reverse transcribed from HepG2 and LFG transfected HepG2 cells. As a positive control we used HTB92 cells, water was used in the negative control. (B) Co-immunoprecipitation of Bax and LFG. HepG2 cells were transfected with pcDNA5/FRT/V5-His-TOPO. Twenty-four-hour transfection cellular extracts including membranes were prepared and mixed with monoclonal antibody directed against V5-tag. pcDNA5/FRT/V5-HIS-CAT transfected cells were used as controls. The eluted protein complexes were separated on SDS-PAGE and visualized as indicated. IP, immunoprecipitates; FL, flowthrough. Lane 1, anti-Bax; lane 2, anti-Bcl-X; lane 3, anti-Bcl-Xs.

signal peptide could not be detected, modifications linked to the secretory pathway were excluded. However, NetPhos and PROSITE predicted several potential phosphorylation sites at the cytoplasmic N-Terminus.

For secondary structure analysis the amino acid sequence was scanned for amino acid clusters, no remarkable ones were detected, however. The computer program coiledcoil detected no probable coiled coil structures pointing to intermembranous protein interaction domains.

LFG belongs to the UPF0005 family. In order to define conserved/functional domains for the antiapoptotic protein LFG we undertook a Prodom analysis with the human amino acid sequence (NP_036438.2) in a search for Pfam and
SMART motifs. The most interesting finding was a conserved C-terminal domain termed UPF0005, a protein family of unknown function (Fig. 4). Due to their conserved C-termini all LFG homologues mentioned in this report show the UPF0005 family signature while the N-termini did not show evolutionarily conserved additional motifs.

RPS-BLAST analysis for the UPF0005 protein family showed that other members of this protein family are distributed through all genetically characterised species including fungi and bacteria. Most interestingly, a better described cytoprotective protein, the anti-apoptotic protein BI-1, possesses the same conserved domain. Other proteins with the UPF0005 motif include the NMDA receptor glutamate binding subunit from Rattus spec., NMDA receptor associated protein from Drosophila melanogaster and NMDA associated glutamat receptor from Danio rerio. Many proteins with the UPF0005 motifs are so far uncharacterised. Among them are the RECS (responsive to centrifugal force and stress) protein from Mus musculus and its human homologue, a growth hormone inducible membrane protein from Mus musculus and a putative aluminum-activated malate transporter from Oryza sativa.

LFG interacts with Bax. $\mathrm{Xu}$ and Reed showed that BI-1 interacts with Bcl-2 but not with Bax (16). To examine whether LFG likewise co-immunoprecipitates with members of the Bcl-2 family, we transfected HepG2 cells, for which no endogenous LFG expression was found by RT-PCR (Fig. 5A), with pcDNA5/FRT/V5-LFG. After $24 \mathrm{~h}$ the cellular extracts were collected and immunoprecipitated using an antibody against the V5-tag. The immunoprecipitates were collected using paramagnetically labelled protein $\mathrm{G}$. The flowthrough was collected and the immunoprecipitates were eluted with boiling Laemmli buffer. Both fractions were analysed by SDS-PAGE followed by immunodetection on Western blotted nitrocellulose membranes (Fig. 5B). While the antiapoptotic members Bcl-X and Bcl-Xs were found exclusively in the flow-through, parts of Bax could be detected in the immunoprecipitates. The main part was found in the flowthrough, however. Vector-transfected controls showed no Bax protein in the precipitate. In order to exclude a crossreaction of Bax to the V5-tag, we performed other control experiments with overexpressed V5-tagged $\mathrm{Cm}$ acetyltransferase (Fig. 5B). There was no Bax in the eluted immunoprecipitates. The results show that Bax is partly retained by co-immunoprecipitation to V5-tagged LFG.

\section{Discussion}

Programmed cell death is a cellular suicide mechanism shared by multicellular organisms and even unicellular forms of life. Beside the characteristic PCD of animals, termed apoptosis, it was found that plants, fungi and even bacteria undergo cellular suicide. Ultrastructural and physiological studies in plants show similarities between PCD in plants and animals (17). The hypersensitive response (HR), a herbal defense to bacteria, fungi and viruses has also been considered as a form of PCD $(18,19)$.

LFG is an evolutionarily conserved protein with regulatory functions in apoptosis. In the present study, we undertook a comprehensive analysis of the primary sequence, which 
showed that the protein topology consists of seven transmembrane domains which are preserved in all examined homologues. Differences are found in the cytoplasmic Ntermini of distantly related species. This might be due to slightly modified cytoprotective functions implicated in the differences between pathways of cell death. The calculated topology predicts a membranous localisation of LFG. Analysis of the primary amino acid sequence in regard to targeting motifs assumes that LFG might localise to the plasma membrane or to the ER. This is in accordance to the findings of Somia et al (6) that overexpressed LFG accumulated in the plasma membrane.

LFG belongs to uncharacterised protein family UPF0005. Another member of this family shares several common features with LFG: the antiapoptotic protein BI-1. The gene encoding for BI-1 was identified in 1994 by Walter et al as a singlecopy gene for which 2 transcripts were found in every organ tested (20). In studies with in vitro translated protein and transiently overexpressed protein, it was shown that BI-1 is localised mainly in the endoplasmatic reticulum (21). The antiapoptotic function of the protein was demonstrated by $\mathrm{Xu}$ and Reed (16) by screening for functional Bax repressors in yeast. Burns and El-Deiry demonstrated BI-1 protection against TRAIL (22). BI-1 expression in human prostate carcinoma and human breast cancer cells linked the antiapoptotic protein to cancer $(23,24)$.

Interestingly, BI-1 homologues also have been found in many different species including Arabidopsis and several other plants (25). Its ability to prevent mammalian Bax-induced cell death in transgenic plants by AtBI-1 has been proved (26). On the other hand BI-1 homologues of oilseed rape and tobacco inhibit Bax-induced cell death in human cells (27). The data collectively show conservation of function of the BI-1 protein in an evolutionary preserved pathway of cytoprotection.

Though the functional mechanism of cell death prevention is largely unknown BI-1 interacts with Bcl-2 and Bcl-XL but not with Bax and Bad when overexpressed in mammalian cells (16). Bcl-2 family proteins play essential roles in the regulation of the intrinsic modus of apoptosis. Accordingly, the apoptosis promoting members Bak and Bax in their active forms are localised in the outer mitochondrial membrane, in a recent report it was shown that they can also be found in the ER (28). Based on studies with BI-1-deficient mice hypersensitive to apoptosis induced by ER stress Chae et al showed that BI-1 regulates an apoptotic pathway linked to ER stress (29). It has been proposed that the membranous protein BI-1 forms ion-conducting channels, or influences the activity of channels from the Bcl-2 family.

Apparently, LFG and BI-1 are closely related cytoprotective proteins. They have a predicted similar topology of a polytopic membrane protein. The amino acid sequence of homologous proteins is evolutionarily conserved within many different species including plants. Although exhaustive genetic and bioinformatic screens have failed to detect plant homologues of the apoptotic machinery, emerging experimental evidence favours the presence of functionally related components of the mammalian apoptosome in higher plants (30).

Though experimental evidence is still missing for LFG, the existence of highly conserved homologues indicates functional conservation for this protein as well. As with BI-1 we showed a connection of LFG to the BCl-2 family. LFG has been shown to protect against Fas-induced apoptosis in mammalian cells (6). Though these are basically different pathways leading to programmed cell death experimental evidence shows that they are interconnected at different levels $(31,32)$. Future investigations on the functional mechanisms of LFG will probably give new insights concerning the signalling pathways of programmed cell death.

\section{Acknowledgements}

This study was supported in part by a grant from the Deutsche Forschungsgemeinschaft (DFG grant no. VO 508/3-1). We are grateful to Andrea Lazaridis for excellent technical assistance.

\section{References}

1. Adams JM and Cory S: Life-or-death decisions by the Bcl-2 protein family. Trends Biochem Sci 26: 61-66, 2001.

2. Deveraux QL and Reed JC: IAP family proteins - suppressors of apoptosis. Genes Dev 13: 239-252, 1999.

3. Goltsev YV, Kovalenko AV, Arnold E, Varfolomeev EE, Brodianskii VM and Wallach D: CASH, a novel caspase homologue with death effector domains. J Biol Chem 272: 19641-19644, 1997

4. Hu S, Vincenz C, Ni J, Gentz R and Dixit VM: I-FLICE, a novel inhibitor of tumor necrosis factor receptor-1- and CD-95induced apoptosis. J Biol Chem 272: 17255-17257, 1997.

5. Inohara N, Koseki T, Hu Y, Chen S and Nunez G: CLARP, a death effector domain-containing protein interacts with caspase- 8 and regulates apoptosis. Proc Natl Acad Sci USA 94: 10717-10722, 1997

6. Somia NV, Schmitt MJ, Vetter DE, Van Antwerp D, Heinemann SF and Verma IM: LFG: an anti-apoptotic gene that provides protection from Fas-mediated cell death. Proc Natl Acad Sci USA 96: 12667-12672, 1999.

7. Schweitzer B, Taylor V, Welcher AA, McClelland M and Suter U: Neural membrane protein 35 (NMP35): a novel member of a gene family which is highly expressed in the adult nervous system. Mol Cell Neurosci 11: 260-273, 1998.

8. Schweitzer B, Suter U and Taylor V: Neural membrane protein 35/Lifeguard is localized at postsynaptic sites and in dendrites. Brain Res Mol Brain Res 107: 47-56, 2002.

9. Wheeler DL, Church DM, Edgar R, et al: Database resources of the National Center for Biotechnology Information: update. Nucleic Acids Res 32: D35-D40, 2004.

10. Kyte J and Doolittle RF: A simple method for displaying the hydropathic character of a protein. J Mol Biol 157: 105-132, 1982.

11. Katoh M and Katoh M: Identification and characterization of human FNBP1L gene in silico. Int J Mol Med 13: 157-162, 2004.

12. Katoh $\mathrm{M}$ and Katoh M: Identification and characterization of human MPP7 gene and mouse Mpp7 gene in silico. Int J Mol Med 13: 333-338, 2004

13. Katoh $\mathrm{M}$ and Katoh M: Identification and characterization of human LLGL4 gene and mouse Llg14 gene in silico. Int J Oncol 24: 737-742, 2004.

14. Katoh $\mathrm{M}$ and Katoh M: Identification and characterization of PDZRN3 and PDZRN4 genes in silico. Int J Mol Med 13: 607-613, 2004.

15. Katoh $\mathrm{M}$ and Katoh M: Identification and characterization of human FHOD3 gene in silico. Int J Mol Med 13: 615-620, 2004.

16. Xu Q and Reed JC: Bax inhibitor-1, a mammalian apoptosis suppressor identified by functional screening in yeast. Mol Cell 1: 337-346, 1998.

17. Gilchrist DG: Programmed cell death in plant disease: the purpose and promise of cellular suicide. Annu Rev Phytopathol 36: 393-414, 1998.

18. Richberg MH, Aviv DH and Dangl JL: Dead cells do tell tales. Curr Opin Plant Biol 1: 480-485, 1998.

19. Ryerson DE and Heath MC: Cleavage of nuclear DNA into oligonucleosomal fragments during cell death induced by fungal infection or by abiotic treatments. Plant Cell 8: 393-402, 1996. 
20. Walter L, Dirks B, Rothermel E, et al: A novel, conserved gene of the rat that is developmentally regulated in the testis. Mamm Genome 5: 216-221, 1994.

21. Cowling RT and Birnboim HC: Preliminary characterization of the protein encoded by human testis-enhanced gene transcript (TEGT). Mol Membr Biol 15: 177-187, 1998.

22. Burns TF and El Deiry WS: Identification of inhibitors of TRAIL-induced death (ITIDs) in the TRAIL-sensitive colon carcinoma cell line SW480 using a genetic approach. J Biol Chem 276: 37879-37886, 2001.

23. Grzmil M, Thelen P, Hemmerlein B, et al: Bax inhibitor-1 is overexpressed in prostate cancer and its specific down-regulation by RNA interference leads to cell death in human prostate carcinoma cells. Am J Pathol 163: 543-552, 2003.

24. Grzmil M, Kaulfuss S, Thelen P, et al: Expression and functional analysis of Bax inhibitor- 1 in human breast cancer cells. J Pathol 208: 340-349, 2006.

25. Chae HJ, Ke N, Kim HR, et al: Evolutionarily conserved cytoprotection provided by Bax Inhibitor-1 homologs from animals, plants, and yeast. Gene 323: 101-113, 2003.
26. Kawai-Yamada M, Jin L, Yoshinaga K, Hirata A and Uchimiya H: Mammalian Bax-induced plant cell death can be down-regulated by overexpression of Arabidopsis Bax Inhibitor-1 (AtBI-1). Proc Natl Acad Sci USA 98: 12295-12300, 2001.

27. Bolduc N, Ouellet M, Pitre F and Brisson LF: Molecular characterization of two plant BI-1 homologues which suppress Baxinduced apoptosis in human 293 cells. Planta 216: 377-386, 2003.

28. Zong WX, Li C, Hatzivassiliou G, et al: Bax and Bak can localize to the endoplasmic reticulum to initiate apoptosis. J Cell Biol 162: 59-69, 2003.

29. Chae HJ, Kim HR, Xu C, et al: BI-1 regulates an apoptosis pathway linked to endoplasmic reticulum stress. Mol Cell 15: 355-366, 2004.

30. Sanchez P, de Torres ZM and Grant M: AtBI-1, a plant homologue of Bax inhibitor-1, suppresses Bax-induced cell death in yeast and is rapidly upregulated during wounding and pathogen challenge. Plant J 21: 393-399, 2000.

31. Roy S and Nicholson DW: Cross-talk in cell death signaling. J Exp Med 192: F21-F25, 2000.

32. Ando Y, Kuroda M, Honda O, et al: The effect of calcium on Fas-mediated apoptosis and secondary necrosis of Jurkat cells. Int J Mol Med 7: 243-247, 2001. 